\title{
Spike-Timing-Dependent Plasticity of Neocortical Excitatory Synapses on Inhibitory Interneurons Depends on Target Cell Type
}

\author{
Jiang-teng Lu, ${ }^{1}$ Cheng-yu Li, ${ }^{1}$ Jian-Ping Zhao, ${ }^{1}$ Mu-ming Poo, ${ }^{1,2}$ and Xiao-hui Zhang ${ }^{1}$ \\ ${ }^{1}$ Institute of Neuroscience and Key Laboratory of Neurobiology, Shanghai Institutes for Biological Sciences, Chinese Academy of Sciences, Shanghai 200031, \\ China, and ${ }^{2}$ Division of Neurobiology, Department of Molecular and Cell Biology, Helen Wills Neuroscience Institute, University of California, Berkeley, \\ California 94720
}

\begin{abstract}
Repetitive correlated spiking can induce long-term potentiation (LTP) and long-term depression (LTD) of many excitatory synapses on glutamatergic neurons, in a manner that depends on the timing of presynaptic and postsynaptic spiking. However, it is mostly unknown whether and how such spike-timing-dependent plasticity (STDP) operates at neocortical excitatory synapses on inhibitory interneurons, which have diverse physiological and morphological characteristics. In this study, we found that these synapses exhibit target-celldependent STDP. In layer 2/3 of the somatosensory cortex, the pyramidal cell (PC) forms divergent synapses on fast spiking (FS) and low-threshold spiking (LTS) interneurons that exhibit short-term synaptic depression and facilitation in response to high-frequency stimulation, respectively. At PC-LTS synapses, repetitive correlated spiking induced LTP or LTD, depending on the timing of presynaptic and postsynaptic spiking. However, regardless of the timing and frequency of spiking, correlated activity induced only LTD at PC-FS synapses. This target-cell-specific STDP was not caused by the difference in the short-term plasticity between these two types of synapses. Activation of postsynaptic NMDA subtype of glutamate receptors (NMDARs) was required for LTP induction at PC-LTS synapses, whereas activation of metabotropic glutamate receptors was required for LTD induction at both PC-LTS and PC-FS synapses. Additional analysis of synaptic currents suggests that LTP and LTD of PC-LTS synapses, but not LTD of PC-FS synapses, involves presynaptic modifications. Such dependence of both the induction and expression of STDP on the type of postsynaptic interneurons may contribute to differential processing and storage of information in cortical local circuits.
\end{abstract}

Key words: synaptic plasticity; spike-timing-dependent plasticity; STDP; excitatory synapse; inhibitory interneuron; target-cell specificity; somatosensory cortex

\section{Introduction}

Repetitive synaptic activity can induce persistent modification of synaptic efficacy in many brain regions in the form of long-term potentiation (LTP) and long-term depression (LTD). Such synaptic plasticity provides a cellular mechanism for experiencedependent refinement of developing neural circuits (Goodman and Shatz, 1993; Song and Abbott, 2001; Zhang and Poo, 2001) and for learning and memory functions of the mature brain (Bliss and Collingridge, 1993; Martin et al., 2000). The precise timing of presynaptic and postsynaptic spiking is often critical for determining whether an excitatory synapse undergoes LTP or LTD.

\footnotetext{
Received Feb. 7, 2007; revised July 12, 2007; accepted July 24, 2007.

This work was supported by National Basic Research Program of China Grant 2006CB806600. M.-M.P. was supported by National Institutes of Health Grant NS36999. X.-H.Z. was supported by a Human Frontier Science Program Career Development Award. We thank S. Zhang, H. Wei. and Q. Hu for technical assistance.

Correspondence should be addressed to either of the following: Xiao-hui Zhang, Institute of Neuroscience, Shanghai Institutes for Biological Sciences, Chinese Academy of Sciences, Shanghai 200031, China, E-mail: xhzhang@ion.ac.cn; or Mu-ming Poo, Department of Molecular and Cell Biology, University of California, Berkeley, CA 94720-3200, E-mail:mpoo@berkeley.edu.

J.P. Zhao's present address: McGovern Institute for Brain Research, Massachusetts Institute of Technology, Cambridge, MA 02139

DOI:10.1523/JNEUROSCI.2513-07.2007

Copyright $\odot 2007$ Society for Neuroscience $\quad$ 0270-6474/07/279711-10\$15.00/0
}

Presynaptic spiking within a few tens of millisecond before postsynaptic spiking ("pre-post") leads to LTP, whereas spiking of the opposite order ("post-pre") leads to LTD (Markram et al., 1997; Bi and Poo, 1998; Debanne et al., 1998; Feldman, 2000; Sjostrom et al., 2001; Froemke and Dan, 2002). Most of our current understanding of such spike-timing-dependent plasticity (STDP) is based on studies of excitatory synapses on principal (glutamatergic) neurons. Whether excitatory synapses on inhibitory (GABAergic) interneurons also exhibit STDP is mostly unknown. The latter synapses serve critical functions in local cortical circuits by initiating feedforward and feedback inhibition.

In the neocortex, inhibitory GABAergic interneurons consist of a diverse population of cell types with distinct morphological, physiological, and molecular characteristics (Markram et al., 2004). This diversity is also reflected in the property of excitatory synapses onto these neurons. In the layer $2 / 3$ of the rat neocortex, pyramidal cell (PC) synapses on bitufted and multipolar interneurons exhibit short-term facilitation and depression, respectively (Reyes et al., 1998). Both the spike-evoked $\mathrm{Ca}^{2+}$ signal and the transmitter release probability of PC synaptic boutons on bitufted interneurons are lower than those on multipolar interneurons (Koester and Johnston, 2005). Whether long-term plas- 
ticity of these synapses also depends on the interneuron type remains unknown. In this study, we have investigated STDP of cortical PC synapses on two interneuron subtypes in layer $2 / 3$ of the somatosensory cortex of juvenile rats, fast spiking (FS) cells and low-threshold spiking (LTS) cells, which were identified by their membrane and firing properties. We found a marked dependence of STDP on the interneuron type: for PC-LTS synapses, LTP and LTD were induced by pre-post and post-pre spike pairing, respectively, whereas only LTD was induced at PC-FS synapses, regardless of the timing and frequency of spiking. Furthermore, we found that LTP and LTD induced by correlated spiking at these synapses were mediated by NMDARs and metabotropic glutamate receptors (mGluRs), respectively. The differences of STDP at these two types of synapses underscore the complexity of activity-induced long-term modification of cortical circuits and suggest potential differential processing and storage of neuronal information through connections mediated by distinct subpopulations of interneurons.

\section{Materials and Methods}

Preparation of cortical slices. Rat somatosensory cortical slices were prepared as described previously (Reyes et al., 1998), with some modifications. Briefly, young Sprague Dawley rats (P13-P16) were anesthetized with sodium pentobarbital $(50 \mathrm{mg} / \mathrm{kg})$ and decapitated. The brain was rapidly dissected and transferred into ice-cold oxygenated artificial CSF [ACSF; composed of (in mM) $124 \mathrm{NaCl}, 2.5 \mathrm{KCl}, 2 \mathrm{MgCl}_{2}, 2 \mathrm{CaCl}_{2}, 1.25$ $\mathrm{NaH}_{2} \mathrm{PO}_{4}, 26 \mathrm{NaHCO}_{3}, 11$ D-glucose, $\mathrm{pH} 7.35, \sim 303 \mathrm{mOsm}$ ] for $2 \mathrm{~min}$. Transverse or parasagittal slices $(300-350 \mu \mathrm{m})$ were prepared with a Vibratome (Vibratome, St. Louis, $\mathrm{MO}$ ) at $0-2^{\circ} \mathrm{C}$ and further incubated with oxygenated ACSF at $34^{\circ} \mathrm{C}$ for $30 \mathrm{~min}$, and then transferred to ACSF at room temperature $\left(25^{\circ} \mathrm{C}\right)$ for $>30 \mathrm{~min}$ before use. For experiments, slices were transferred to the recording chamber and perfused with oxygenated ACSF at $32-34^{\circ} \mathrm{C}$. The care and use of rats followed the animal use guidelines of Shanghai Institutes for Biological Sciences, Chinese Academy of Sciences (Shanghai, China).

Electrophysiology. Simultaneous dual (or triple) whole-cell recordings (in current clamp) from PCs and interneurons in layer 2/3 were made with Axopatch 700A or 200B amplifiers (Molecular Devices, Union City, CA) using an upright microscope (BX51; Olympus, Tokyo, Japan) equipped with differential interference contrast (DIC) optics and infrared video camera. The internal solution of the recording pipette contained (in $\mathrm{mM}$ ) $130 \mathrm{~K}$-gluconate, $20 \mathrm{KCl}, 10 \mathrm{Na}$-phosphocreatine, 10 HEPES, 4 ATP-Mg, 0.3 GTP, and 0.2 EGTA, adjusted to pH 7.3 with $\mathrm{KOH}$ and to $\sim 298 \mathrm{mOsm}$. The pipette resistance was 4-6 M $\Omega$. To assay the synaptic efficacy of PC-interneuron connections, EPSPs were recorded from postsynaptic interneurons when spikes were elicited in the presynaptic PC by the injection of current pulses (3 ms duration, 1-2 nA) at a low frequency $(0.033 \mathrm{~Hz})$. Signals were recorded and filtered at 2 $\mathrm{kHz}$, digitized at $10 \mathrm{kHz}$ (Digidata 1322A; Molecular Devices) and further analyzed using the pClamp 9.0 software (Molecular Devices). Correlated presynaptic and postsynaptic spiking at various time intervals was used to induce persistent synaptic modification. During the induction, postsynaptic spiking was evoked by injection of depolarizing current pulses (1-2 nA, $3 \mathrm{~ms}$ ). In experiments on the STDP time window, presynaptic and postsynaptic spike timing were defined as the onset of the EPSP and the peak of the spike, respectively. The stable baseline of EPSPs was obtained for $10 \mathrm{~min}$ before the application of correlated spiking, and the postinduction mean EPSPs were measured for a period between 10 min after correlated spiking and the end of recording (normally 30-60 min after the correlated spiking). Percentage values of the mean EPSP amplitude were determined relative to the mean baseline amplitude before spike pairing. Those EPSPs with amplitudes below the noise level were regarded as transmission failures, which were included in the calculation of mean EPSPs as events with the amplitude of $0 \mathrm{mV}$. Input resistance of presynaptic and postsynaptic neurons was monitored throughout the recording by the injection of a hyperpolarizing current $(-40 \mathrm{pA}, 300 \mathrm{~ms}, 0.033 \mathrm{~Hz})$. Data were accepted for analysis only for cases in which the input resistance varied $<30 \%$ throughout the experiment. Data are presented as mean \pm SEM. The comparison of results before and after the experimental treatment was done by paired $t$ test and that between results for different treatments was done by unpaired $t$ test. Cumulative distribution was analyzed by Kolmogorov-Smirnov test. All chemicals were from Sigma (St. Louis, MO) except mGluR antagonists $(R S)$ - $\alpha$-methyl-4-carboxyphenylglycine (MCPG), LY367385 [( $S)-(+)-$ $\alpha$-amino-4-carboxy-2-methylbenzeneacetic acid], and MEPS [2-methyl6-(phenylethynyl)-pyridine hydrochloride]; NMDAR antagonist (-)-5methyl-10,11-dihydro-5H-dibenzo[a,d]cyclohepten-5,10-imine maleate (MK801); and CB1 receptor antagonist $N$-(piperidin-1-yl)-5-(4-iodophenyl)1-(2,4-dichlorophenyl)-4-methyl-1 H-pyrazde-3-carboxamide (AM251), which were from Tocris (Bristol, UK).

Reconstruction of neuronal morphology. Slices containing interneurons that were physiologically identified and filled with biocytin $(0.2 \%)$ through the recording pipette were incubated at $4^{\circ} \mathrm{C}$ for $4-7 \mathrm{~d}$ with $4 \%$ paraformaldehyde in PBS, $\mathrm{pH}=7.4$. After the fixation, slices were rinsed in PBS (5 min for three times), quenched $\left(0.3 \% \mathrm{H}_{2} \mathrm{O}_{2} / \mathrm{PBS}\right.$ for $\left.30 \mathrm{~min}\right)$, rinsed again in PBS, and then exposed to avidin-biotin horseradish peroxidase complex containing 0.3\% Triton X-100 (ABC kit; Vector Laboratories, Burlingame, $\mathrm{CA}$ ) for $4 \mathrm{~d}$. On the fourth day, slices were rinsed again in PBS, exposed to the 3,3'-diaminobenzidine (DAB) staining reaction for 3-10 min with DAB kit (Vector Laboratories), and then dehydrated in a series of ethanol solutions of incremental concentrations ( 75 , 95 , and $100 \%$ ) and cleared in dimethylbenzene ( 5 min for three times) before mounting into slides. Three-dimensional light-microscopic reconstruction of dendritic and axonal arbors of DAB-stained neurons was performed using Neurolucida system (MicroBrightField, Williston, VT).

\section{Results}

Identification of fast-spiking and low-threshold-spiking cells Dual whole-cell recordings were made from synaptically connected pairs of layer $2 / 3$ neurons in acutely isolated somatosensory cortical slices from P13-P16 rats. The PCs and inhibitory interneurons were identified by their morphology and firing patterns: PCs have clear apical dendrites and low firing rates with adaptation, whereas inhibitory interneurons lack apical dendrites and can sustain prolonged spiking at a high rate. We focused on two distinct interneuron classes, FS and LTS cells in layer 2/3, identified by their membrane properties and firing patterns, including input resistance, spiking threshold, spike frequency adaptation, spike duration, and amplitude of afterhyperpolarization (Fig. 1A, $B$, Table 1). The differences in membrane and firing properties we observed for layer 2/3 FS and LTS cells were similar to those reported in cortical layer 4/5 (Kawaguchi and Kubota, 1997; Bacci et al., 2003). Morphological reconstruction of biocytin-filled cells (six LTS cells and eight FS cells) after the recording further revealed that layer 2/3 FS cells typically had a dense axonal plexus, multiple axons/dendrites extended radially from their cell body, whereas LTS cells were characterized by less pronounced perisomatic distribution of axonal and dendritic arbors, but extensive axonal and dendritic arborization out of layer 2/3 (Fig. 1 $A, B$ ). Compared with previously identified layer 2/3 multipolar and bitufted cells, which were distinguished by the distinct shape of their somata and the location/number of proximal dendrites under infrared-DIC optics or after reconstruction of dye-filled cells (Reyes et al., 1998; Koester and Johnston, 2005), all FS cells identified in the present study resembled multipolar cells, whereas LTS cells we identified consisted of mostly bitufted cells, with a few cells exhibiting multipolar morphology.

Dual whole-cell recordings of EPSPs showed that PCs made excitatory inputs to both FS and LTS cells (Fig. 1C,D). The PCLTS synapses exhibited lower synaptic efficacy and higher rate of transmission failure than PC-FS synapses. We also consistently 

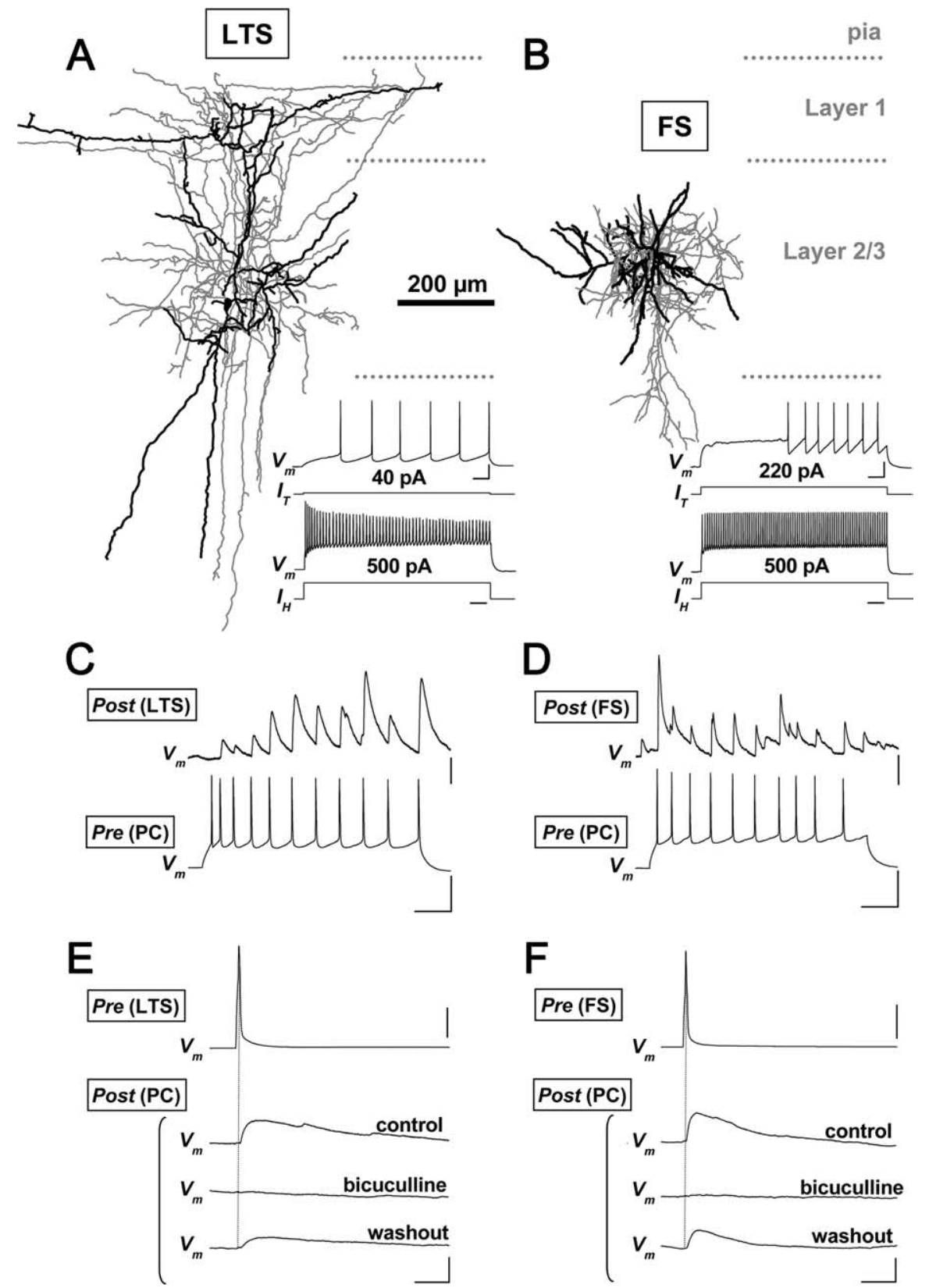

Figure 1. Identification of synaptic connections between $P C$ and LTS or FS interneurons in layer $2 / 3$ of the somatosensory cortical slices. $A, B$, Firing property and morphology of LTS and FS interneurons. Reconstruction of a typical biocytin-filled LTS $(\boldsymbol{A})$ or FS ( $\boldsymbol{B}$ ) cell in layer 2/3 in rat somatosensory cortex (soma and dendrites in black, and axons in gray). Insets, Example recordings depicting the firing characteristics of the corresponding cell in response to a step depolarizing current. The threshold current $\left(I_{T}\right)$ for initiating APs was $\sim 40$ and $\sim 220 \mathrm{pA}$ for the LTS cell and the FS cell, respectively (top traces). At a higher current step $\left(I_{H}, 500 \mathrm{pA}\right)$ that induced high-frequency spiking, the LTS cell exhibited more apparent frequency adaptation than the FS cell (bottom traces) Calibration: $20 \mathrm{mV}, 50 \mathrm{~ms}$. C, D, Example recordings showing short-term facilitation of PC-LTS synapses $(\boldsymbol{C})$ and depression at PC-FS synapses $(\boldsymbol{D})$ when a train of APs was elicited in the presynaptic PC. Calibration: top traces, $2 \mathrm{mV}, 100 \mathrm{~ms}$; bottom traces, 40 $\mathrm{mV}, 100 \mathrm{~ms}$. $\boldsymbol{E}, \boldsymbol{F}$, Example recordings showing reciprocal IPSPs recorded from the PCs when single APs were evoked in the LTS (E) or FS $(\boldsymbol{F})$ cell. Bath application of the $\mathrm{GABA}_{\mathrm{A}}$ receptor antagonist bicuculline $(5 \mu \mathrm{M})$ reversibly abolished the IPSPs. Calibration: top $40 \mathrm{mV}, 25 \mathrm{~ms}$; bottom, $2 \mathrm{mV}, 25 \mathrm{~ms}$.

observed paired-pulse facilitation at PC-LTS synapses and paired-pulse depression at PC-FS synapses (Table 2), in agreement with the previous finding on layer 2/3 PC-interneuron synapses (Reyes et al., 1998; Koester and Johnston, 2005). Among those interconnected cell pairs, PC/FS pairs had more frequent reciprocal connections than PC/LTS pairs (Table 2). The reciprocal connections were revealed by IPSPs recorded in the PC in response to stimulation of the interneuron. These IPSPs had a long decay time and were susceptible to reversible blockade by bath-applied bicuculline methiodide, a competitive $\mathrm{GABA}_{\mathrm{A}}$ receptor antagonist (Fig. 1E,F).

\section{STDP at PC-LTS and PC-FS synapses}

Excitatory synapses on neocortical PCs are known to exhibit STDP (Markram et al., 1997; Feldman, 2000; Sjostrom et al., 2001; Froemke and Dan, 2002). Using paired recording in somatosensory cortical slices, we found a marked target-cell-dependent STDP at PC-interneuron synapses. At PCLTS synapses, repetitive correlated presynaptic and postsynaptic spiking ( 12 trains at $5 \mathrm{~s}$ intervals, with five spikes at $20 \mathrm{~Hz}$ in each train) induced a persistent synaptic potentiation when presynaptic spikes preceded postsynaptic spikes by $+8 \mathrm{~ms}$ (prepost) (Fig. $2 \mathrm{~A}$ ), but a persistent depression when the order of spiking was reversed ( $-8 \mathrm{~ms}$; post-pre) (Fig. $2 \mathrm{~B})$. In contrast, the same spiking protocols induced persistent depression of PC-FS synapses, regardless of the order of spiking (Fig. 2D,E). There was no difference in the magnitude of LTD between the two data sets from the +8 and $-8 \mathrm{~ms}$ pairing experiments (supplemental Fig. 1A, available at www. jneurosci.org as supplemental material). Summary of all experiments are shown in Figure 2, $C$ and $F$. Consistent with a narrow STDP window for the induction of synaptic modification at neocortical excitatory synapses (Dan and Poo, 2006), we found that correlated spiking at $20 \mathrm{~Hz}$ with spike pairing interval of either $+25 \mathrm{~ms}$ (pre-post) or $-25 \mathrm{~ms}$ (post-pre), which are essentially equivalent at this spiking frequency, had no effect on synaptic efficacy (Fig. 2C,F). These results suggest the existence of target-cell-specific STDP of PC-interneuron synapses in layer $2 / 3$ of the somatosensory cortex.

\section{Triplet recordings of spike-timing- dependent LTP and LTD}

To exclude the possibility that the apparent target-cell-specific STDP described above was the result of different types of PCs making selective innervation on LTS versus FS cells, we further performed experiments using simultaneous whole-cell recordings from a triplet of neurons, consisting of one PC innervating both a FS cell and an LTS cell. In the example recording shown in Figure $3 A$, a train of spikes in the PC evoked EPSPs that exhibited synaptic facilitation in the LTS cell, but synaptic depression in the FS cell. After the same repetitive correlated pre-post spiking at +8 ms was applied simultaneously to the PC-LTS and PC-FS synapses, we observed LTP of PC-LTS synapses, but LTD of PC-FS synapses (Fig. 3B). Such opposite 
Table 1. Properties of FS and LTS interneurons

\begin{tabular}{|c|c|c|}
\hline & $\mathrm{FS}(n)$ & $\operatorname{LTS}(n)$ \\
\hline Membrane resistance (M $\Omega$ ) & $94.3 \pm 4.7$ & $399.6 \pm 28.6(30)$ \\
\hline Spike half-width (ms) & $0.85 \pm 0.02(31)$ & $1.36 \pm 0.08(30)$ \\
\hline AHP amplitude (mV) ${ }^{a}$ & $23.1 \pm 0.6$ & $14.1 \pm 0.6$ \\
\hline Threshold current $(\mathrm{pA})^{b, f}$ & $269 \pm 17$ & $43 \pm 3$ \\
\hline Onset frequency $(\mathrm{Hz})^{c}$ & $26.6 \pm 1.5$ & $11.7 \pm 1.4$ \\
\hline I/O slope $(\mathrm{Hz} / \mathrm{pA})^{d, f}$ & $0.30 \pm 0.01(21)$ & $0.09 \pm 0.01(19)$ \\
\hline Spike frequency adaptation ${ }^{e, f}$ & $1.07 \pm 0.01(21)$ & $2.05 \pm 0.20(19)$ \\
\hline
\end{tabular}

Membrane characteristics and firing properties of FS and LTS cells in layer $2 / 3$ of somatosensory cortex. $n$, Number of cells examined ( $p<0.001$ for all parameters, Student's $t$ test).

${ }^{a}$ Afterhyperpolarization (AHP), calculated as the difference between the action potential threshold and the most negative level reached during the repolarizing phase.

${ }^{b}$ Current injections with duration $600 \mathrm{~ms}$ in $20 \mathrm{pA}$ steps from 0 to $600 \mathrm{pA}$. The threshold current was the amplitude of the first step current to evoke action potentials.

'Onset frequency was the spike frequency of the action potential trains elicited by the threshold current.

${ }^{\mathrm{d}}$ The $\mathrm{I} / 0$ slope is the slope of the input (injected current) minus output (firing frequency) curve, with the amplitude of the injected currents ranging from 300 to $600 \mathrm{pA}$.

eSpike-frequency adaptation, calculated as the mean of the ratios of the instantaneous frequencies at the fourth and the last spike intervals in every action potential train elicited by step-current injections from 300 to $600 \mathrm{pA}$.

${ }^{f}$ Ranges completely nonoverlapping.

Table 2. Properties of layer 2/3 PC-interneuron synaptic connections

\begin{tabular}{lcc}
\hline & PC-FS $(n)$ & PC-LTS $(n)$ \\
\hline Mean EPSP amplitude (mV) & $2.95 \pm 0.22(142)$ & $0.72 \pm 0.08(95)$ \\
Failure rate (\%) & $5.3 \pm 0.8(142)$ & $48.9 \pm 3.0(95)$ \\
Paired-pulse ratio (\%) $^{a}$ & $76.1 \pm 2.9(43)$ & $119 \pm 12 \quad(28)$ \\
Reciprocal connections (\%) $^{b}$ & $59.9(97 / 162)$ & $21.3(32 / 150)$ \\
\hline
\end{tabular}

$n$, Number of cell pairs examined.

${ }^{a}$ Paired-pulse ratio was determined as the ratio of the amplitude of the first and the second EPSP evoked by two consecutive spikes in the $\mathrm{PC}$ at an interval of $100 \mathrm{~ms}$.

${ }^{b}$ Reciprocal connections refer to cell pairs that were synaptically interconnected.

synaptic modifications by the same spiking activity at two different $\mathrm{PC}$-interneuron connections were observed in all five triplets for which successful long-term recording was made (Fig. 3C). These results strongly support the notion of target-cell-specific STDP of PC-interneuron synapses in neocortical layer $2 / 3$.

\section{Target-cell-specific STDP is not caused by differential short-term plasticity}

A train of five spikes at $20 \mathrm{~Hz}$ of presynaptic PC elicited a series of EPSPs with increasing amplitudes at PC-LTS synapses, but decreasing amplitudes at PC-FS synapses, as a result of short-term synaptic facilitation and depression, respectively (Fig. 4A,B). This target-cell-dependent short-term modification of synaptic efficacy was clearly observed during the induction of LTP/LTD using correlated spiking at $20 \mathrm{~Hz}$ (Fig. $2 \mathrm{~A}, \mathrm{D}$ ), leaving the possibility that target-cell-specific STDP of PC-interneuron synapses observed at $20 \mathrm{~Hz}$ may be attributable to the difference in shortterm plasticity. Consistent with the known dependence of shortterm plasticity on the spiking interval (Zucker and Regehr, 2002), we found that five consecutive spikes in presynaptic PC at $1 \mathrm{~Hz}$ did not result in any synaptic facilitation/depression at either PC-LTS or PC-FS synapses (Fig. 4A, B). Nevertheless, we found that correlated spiking at $1 \mathrm{~Hz}$ (at $+8 \mathrm{~ms}$ interval, 60 pulses) induced LTP at PC-LTS synapses, but LTD at PC-FS synapses (Fig. 4C), similar to that induced by correlated spiking at $20 \mathrm{~Hz}$ (Fig. 2). Thus, the target-cell-specific STDP of PC-interneuron synapses is not caused by differences in short-term synaptic plasticity. The short- and long-term forms of activity-dependent synaptic plasticity, although both depend on the target cell identity, are apparently mediated by distinct cellular mechanisms.

\section{Dependence of PC-FS synaptic modification on spiking frequency and timing}

The absence of LTP at PC-FS synapses after correlated pre-post spiking ( $+8 \mathrm{~ms}$ interval, at 20 or $1 \mathrm{~Hz})$ may be attributable to a distinct dependence on the frequency or timing of spiking for LTP induction at these synapses. When a wide range of spiking frequencies (with the same total number of spikes) were tested, we observed robust LTD when correlated pre-post spiking (at +8 ms) was applied at 1,5 , and $20 \mathrm{~Hz}$, but no changes in synaptic efficacy at 0.25 and $40 \mathrm{~Hz}$ (Fig. 5A). Furthermore, when a wide range of spike intervals between $\pm 100 \mathrm{~ms}$ was examined with the spiking frequency set at $1 \mathrm{~Hz}$, we found a symmetric time window, with significant LTD induced within a window between about $\pm 25 \mathrm{~ms}$ (Fig. $5 B$ ). Together, these results indicate the propensity for PC-FS synapses for spike-timing-dependent LTD, regardless of the temporal order of spiking.

\section{Induction mechanisms underlying spike-timing-dependent LTP/LTD}

Additional studies were performed to examine the induction mechanism underlying STDP at these two types of PC-interneuron synapses. We first examined the involvement of NMDARs, which are primarily responsible for the postsynaptic $\mathrm{Ca}^{2+}$ elevation required in both LTP and LTD of many excitatory synapses on glutamatergic neurons (Malenka and Bear, 2004; Dan and Poo, 2006). When NMDAR blocker $\mathrm{D}(-)$-2-amino-phosphonovaleric acid (D-AP5; $50 \mu \mathrm{M}$ ) was added to the bath solution $30 \mathrm{~min}$ before the recording and kept throughout the experiment, we found that prepost spike pairing (at $+8 \mathrm{~ms}$ ) failed to induce LTP at PC-LTS synapses, but induced LTD instead, suggesting that NMDAR activation (induced by spike pairing at $+8 \mathrm{~ms}$ ) (Fig. $6 A$ ) may have "masked" or "inhibited" LTD induction. Postsynaptic NMDAR activation was required for the LTP induction at the PC-LTS synapses, because selective postsynaptic loading of NMDAR open-channel blocker MK801 (1 mM in the internal solution) into the LTS cell resulted in the induction of LTD instead of LTP after pre-post ( $+8 \mathrm{~ms})$ spike pairing (Fig. 6A). Under either the AP5 or MK801 treatment, the magnitude of "unmasked" LTD was similar to that of normal LTD induced at post-pre $(-8 \mathrm{~ms})$ spike pairing $(p=0.46$ and 0.38 for AP5 ands MK801 treatments, respectively, Kolmogorov-Smirnov test). In contrast to the dependence of LTP on NMDARs, induction of LTD by post-pre ( $-8 \mathrm{~ms})$ spike pairing at PC-LTS synapses was unaffected by bath-applied AP5 (Fig. 6C). At PC-FS synapses, blocking NMDARs with similar bath-applied AP5 also had no effect on the induction of LTD by either pre-post or post-pre spike pairing (Fig. 6D). Thus, LTP induction at PC-LTS synapses requires the activation of postsynaptic NMDARs, whereas LTD induction at both PC-interneuron synapses does not involve NMDAR activation.

Because activation of mGluRs is known to be required for LTD induction at several other neocortical synapses (Egger et al., 1999; Bender et al., 2006), we further examined whether LTD induction at these PC-interneuron synapses depends on the activation of mGluRs. We found that bath application of mGluR competitive antagonist MCPG (0.5 mM) indeed prevented LTD induction by correlated spiking at both PC-LTS (Fig. 6C) and PC-FS (Fig. 6D) synapses, but had no effect on LTP induction at PC-LTS synapses (Fig. 6B). Additional pharmacological experiments suggested that activation of mGluRla receptor and inositol 1,4,5-triphosphate $\left(\mathrm{IP}_{3}\right)$ receptor-mediated $\mathrm{Ca}^{2+}$ elevation in FS cells was required for the induction of LTD at PC-FS synapse (supplemental Fig. 2, available at www.jneurosci.org as supplemental material). Interestingly, MCPG also effectively blocked 

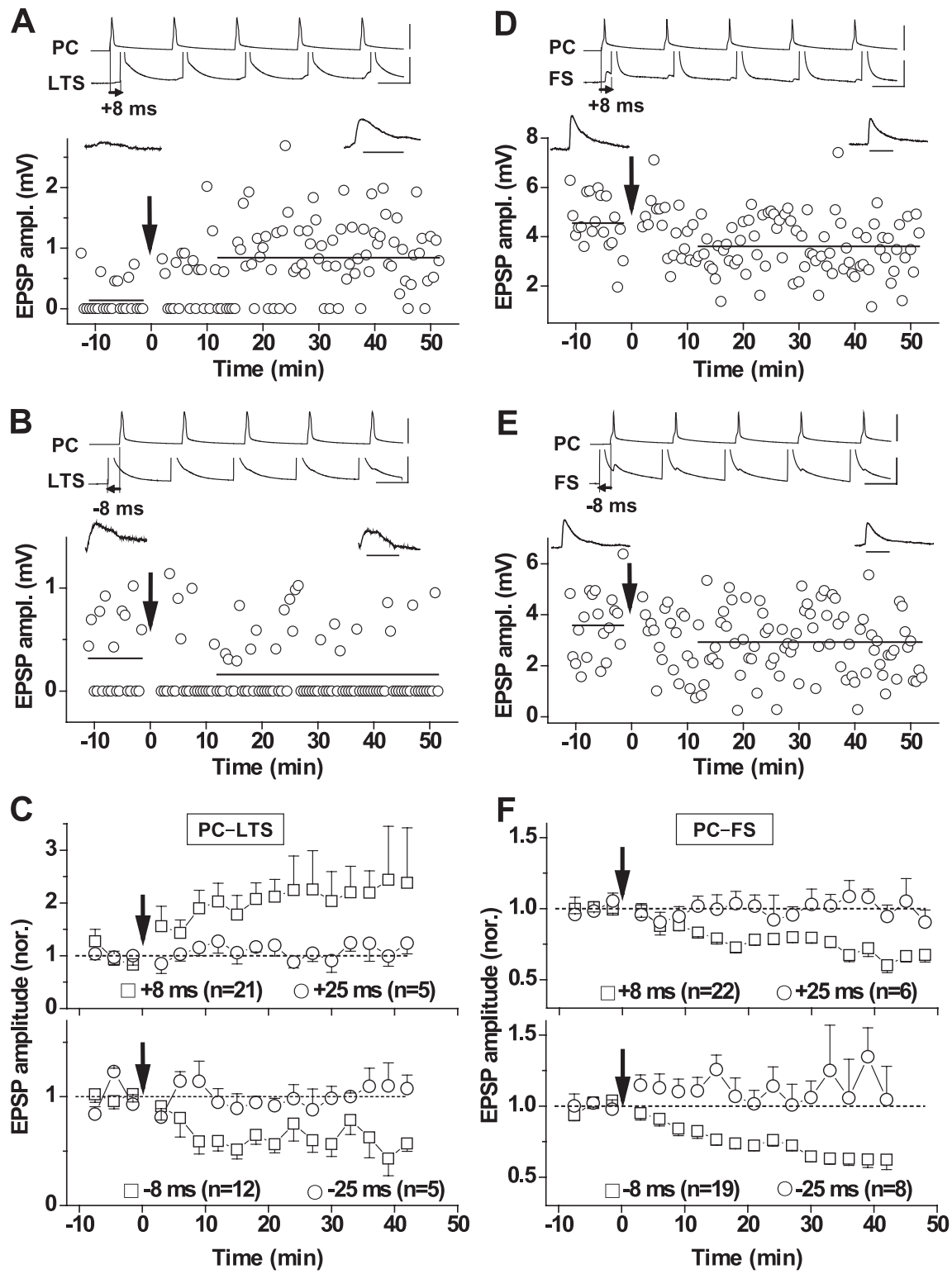

Figure 2. Distinct spike-timing-dependent LTP/LTD at PC-LTS and PC-FS synapses. $A, B$, Example recordings from PC-LTS cell pairs showing persistent increase $(\boldsymbol{A})$ and decrease $(\boldsymbol{B})$ in the EPSP amplitude after repetitive correlated presynaptic and postsynaptic spiking (at $t=0 \mathrm{~min}$; arrow) at intervals of $+8 \mathrm{~ms}$ ( $\boldsymbol{A}$; pre-post pairing) and $-8 \mathrm{~ms}$ ( $\boldsymbol{B}$; post-pre pairing), respectively. Top traces, Example recordings of membrane potential changes in the presynaptic $\mathrm{PC}$ and postsynaptic LTS during spike pairing at 20 $\mathrm{Hz}$; the postsynaptic spikes were truncated. Calibration: PC traces, $100 \mathrm{mV}, 25 \mathrm{~ms}$; LTS traces, $12.5 \mathrm{mV}, 25 \mathrm{~ms}$. Horizontal lines indicate the mean value (including failures) over the duration covered by the line. EPSP traces above depict averaged EPSP over the duration indicated by the black line, with failure included as $0 \mathrm{mV}$. Calibration: $20 \mathrm{~ms}$. D, E, Similar to that in $\boldsymbol{A}, \boldsymbol{B}$, except that the recordings were from PC-FS cell pairs. Persistent synaptic depression was induced after spike pairing at either $+8 \mathrm{~ms}$ or $-8 \mathrm{~ms}$ intervals. $C, F$, Summary of results from all experiments similar to that described in $A, B, D, E$, including data using spiking intervals of +25 and $-25 \mathrm{~ms}$. The data points represent mean EPSP amplitude over a $3 \mathrm{~min}$ bin, normalized by the mean value before the induction. $n$, Number of cell pairs examined. For the induction at $\pm 25 \mathrm{~ms}$, no significant change in the EPSP amplitude was observed for all cases ( $p>0.1$, paired $t$ test). Error bars indicate SEM.

the induction of LTD at PC-LTS synapses by pre-post spiking $(+8$ $\mathrm{ms}$ ) in the presence of AP5 (Fig. $6 \mathrm{~A}$ ), suggesting that the activation of mGluR is essential for LTD induction at PC-interneuron synapses. These results are consistent with the notion that NMDAR and mGluR serve as two independent coincidence detectors for the induction of spike-timing-dependent LTP and LTD, respectively.

At neocortical excitatory synapses on PCs, spike-timingdependent LTD requires retrograde signaling by endocannabinoid (Sjostrom et al., 2003; Bender et al., 2006). However, we found that the bath application of a selective antagonist of cannabinoid type 1 (CB1) receptor, AM251 $(1 \mu \mathrm{M})$ did not affect the induction of spike-timingdependent LTP (Fig. 6B) and LTD (Fig. $6 C)$ at PC-LTS synapses. The LTD induced by pre-post spiking in the presence of AP5 was also not affected by AM251 (Fig. 6A). Similarly, AM251 treatment did not affect LTD induction at PC-FS neurons by either pre-post or post-pre spiking (Fig. 6D). We note that the AM251 treatment used here was effective in blocking the endocannabinoid-mediated slow hyperpolarization (self-inhibition) after the burst spiking in LTS cells (data not shown), similar to that found in layer 4 LTS cells (Bacci et al., 2004). Thus, mGluR-dependent LTD of these PC-interneuron synapses differs from that of excitatory synapses on PCs by its independence of endocannabinoid signaling.

\section{A lower proportion of NMDARs at PC-FS synapses}

The above results suggest a dominant role of NMDAR signaling in the induction of spike-timing-dependent LTP at PC-interneuron synapse. Additional studies were performed to examine postsynaptic differences at PC-FS and PC-LTS synapses that may contribute to differential STDP. The EPSCs mediated by NMDARs and nonNMDARs at PC-LTS and PC-FS synapses were measured in a medium containing no $\mathrm{Mg}^{2+}$. The non-NMDAR-mediated component was defined as the EPSC observed after bath application of D-AP5 $(50 \mu \mathrm{M})$, and this component could be largely blocked by CNQX (6-cyano-7-nitroquinoxaline2,3-dione, $10 \mu \mathrm{M}$ ) (supplemental Fig. 3A, available at www.jneurosci.org as supplemental material). The NMDAR-mediated component was obtained by subtracting the total EPSC with the non-NMDAR-mediated component. To estimate the relative amount of NMDARs and non-NMDARs at these synapses, we calculated the integrated charge associated with the each EPSC component. As shown in supplemental Fig. $3 B$ (available at www.jneurosci.org as supplemental material), there was no significant difference in the distribution and the mean of integrated EPSC charges between two types of synapses. However, when the charge ratio for NMDAR-EPSC versus total EPSC (NMDAR/ total) was calculated for each type of connection, we found that the ratio for PC-FS synapses $(0.26 \pm 0.06 ; n=7)$ was $\sim 33 \%$ of that of PC-LTS synapses $(0.79 \pm 0.26 ; n=8)$ (supplemental Fig. $3 C$, available at www.jneurosci.org as supplemental material). Whether this lower level of NMDARs relative to non-NMDARs at PC-FS synapses contributes to the absence of NMDARdependent LTP induction described above remains to be further investigated. 

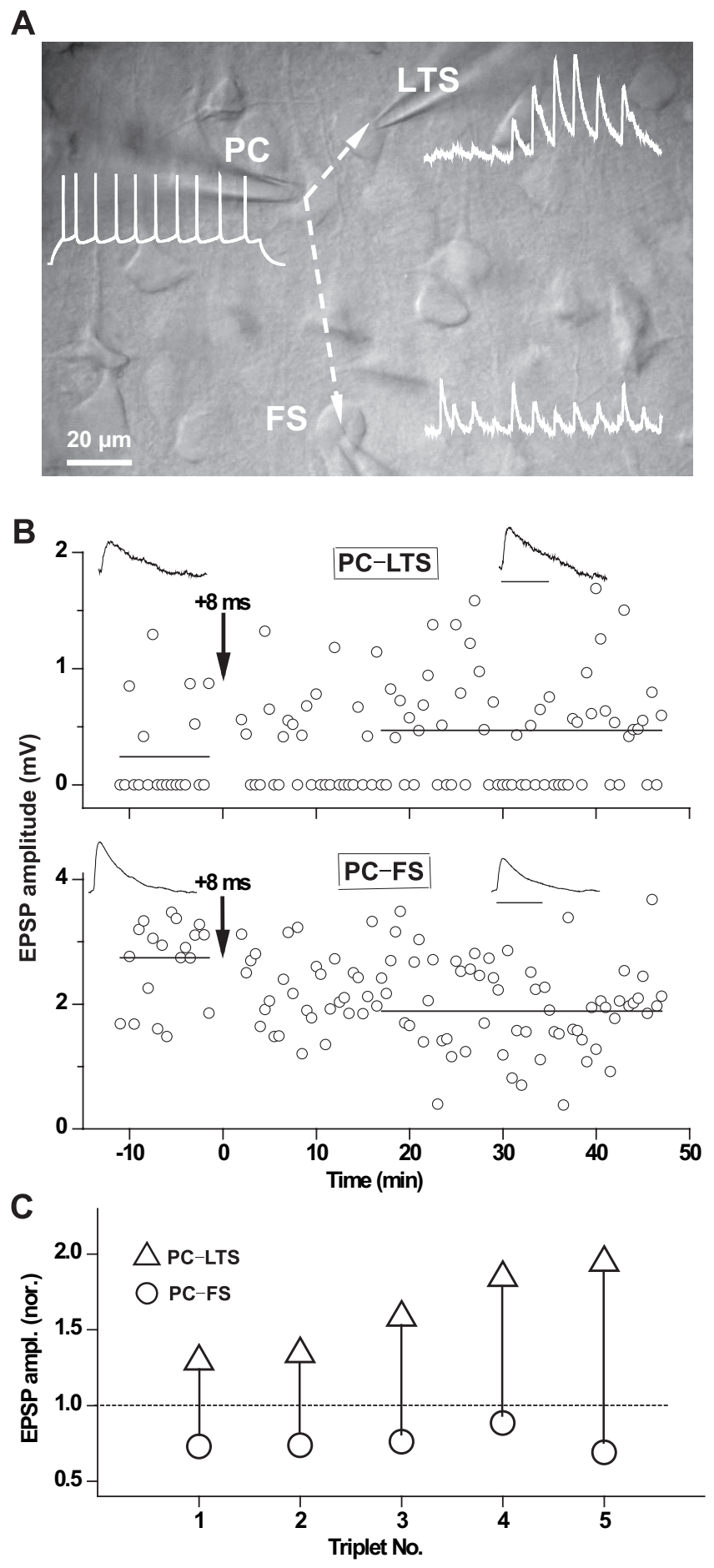

Figure 3. Triplet recordings showed target-cell-specific STDP of divergent synapses made by the same PC on LTS and FS cells. $A$, An image of a representative triplet (i.e., one PC innervating one LTS cell and one FS cell, depicted by the dashed lines) in the layer $2 / 3$ of the somatosensory cortical slice. White traces shown near the recorded cells depict a train of APs evoked in the PC by current injection and corresponding EPSPs evoked in the LTS or FS cell. $\boldsymbol{B}$, Example recordings from the triplet shown in A: LTP ( $194 \%$ of the baseline, for data beyond 15 min after induction; top) at PC-LTS synapses and LTD (69\% of the baseline; bottom) at PC-FS synapses, induced by the same repetitive correlated pre-post ( $+8 \mathrm{~ms}$ ) spike pairing (arrow). Horizontal lines indicate the mean value (including failures) over the duration covered by the line. Traces above depict averaged EPSPs over the duration indicated by the line. Calibration: $20 \mathrm{~ms}$. C, Results from five triplet experiments similar to that shown in $\boldsymbol{B}$ (PC-LTS synapses, $160 \pm 13 \% ; \mathrm{PC}-\mathrm{LTS}$ synapses, $76 \pm 3 \%$, relative to the baseline before induction). Data from the same triplet are connected by the line.
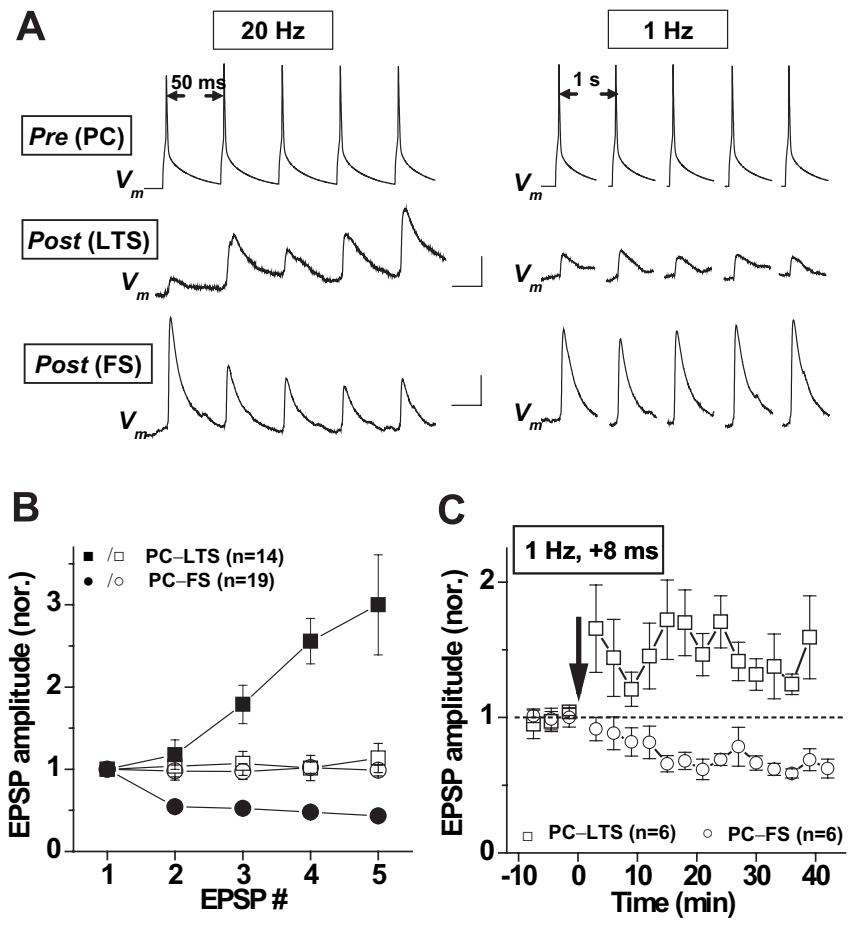

Figure 4. Target-cell-specific STDP is independent of short-term synaptic plasticity. A, EXamples of EPSPs evoked by the a train of five presynaptic spikes at 20 and $1 \mathrm{~Hz}$ at PC-LTS and PC-FS synapses. Traces were the average of 60 EPSPs. Calibration: $1 \mathrm{mV}, 25 \mathrm{~ms}$. $\boldsymbol{B}$, Changes in the EPSP amplitude elicited by five spikes. Summary of results from all experiments shown in $A$. The amplitude of EPSPs was normalized by that of the first EPSP. Filled symbols, Spiking at $20 \mathrm{~Hz}$; open symbols, spiking at $1 \mathrm{~Hz}$. Error bars depict SEM. C, Time course of the change in EPSP amplitude before and after the $1 \mathrm{~Hz}$ pre-post spike pairing ( $+8 \mathrm{~ms}, 60$ pulses) at PC-LTS (square) and PC-FS (circle) synapses. The amplitude of EPSPs was normalized by the mean value before spike pairing. $n$, Number of cell pairs examined.

\section{Expression of STDP is also target-cell-dependent}

In addition to the induction mechanism, we have investigated whether STDP is similarly expressed at PC-LTS and PC-FS synapses by examining the properties of synaptic transmission before and after induction of LTP/LTD by spike pairing. The change in the variance of EPSP amplitudes [quantified by the coefficient of variation $(\mathrm{CV})]$ has often been used to suggest presynaptic modification. Enhanced presynaptic neurotransmitter release, caused by an increase in the number of release sites and/or the probability of release, will result in a reduction in CV (Bekkers and Stevens, 1990; Malinow and Tsien, 1990), although changes in CV may also result from the activation of postsynaptic silent AMPAR clusters (Liao et al., 1995) or an uneven distribution of the loci (proximal vs distal sites) that undergo changes in synaptic efficacy (Sakmann et al., 1998). We found that after the induction of LTP and LTD at PC-LTS synapses, there was a decrease and an increase in the average CV of evoked EPSPs, respectively (Fig. $7 A)$. In addition, transmission at these synapses also showed bidirectional changes in the mean failure rate, with a reduced rate after LTP ( $69 \pm 7 \%$ of the control; $n=21 ; p=0.002$; paired $t$ test $)$ and an increased rate after LTD ( $229 \pm 48 \%$ of the control; $n=8$; $p=0.02$; paired $t$ test) (Fig. $7 B$ ). These findings of bidirectional changes in the CV and failure rate strongly suggest that STDP of PC-LTS synapses is expressed as presynaptic modification of transmitter release. Interestingly, when PC-LTS synapses underwent LTD after LTP-inducing pairing protocol $(+8 \mathrm{~ms})$ in the presence of AP5, we observed changes in the $\mathrm{CV}$ and failure rate similar to those found for LTD induced by $-8 \mathrm{~ms}$ pairing proto- 

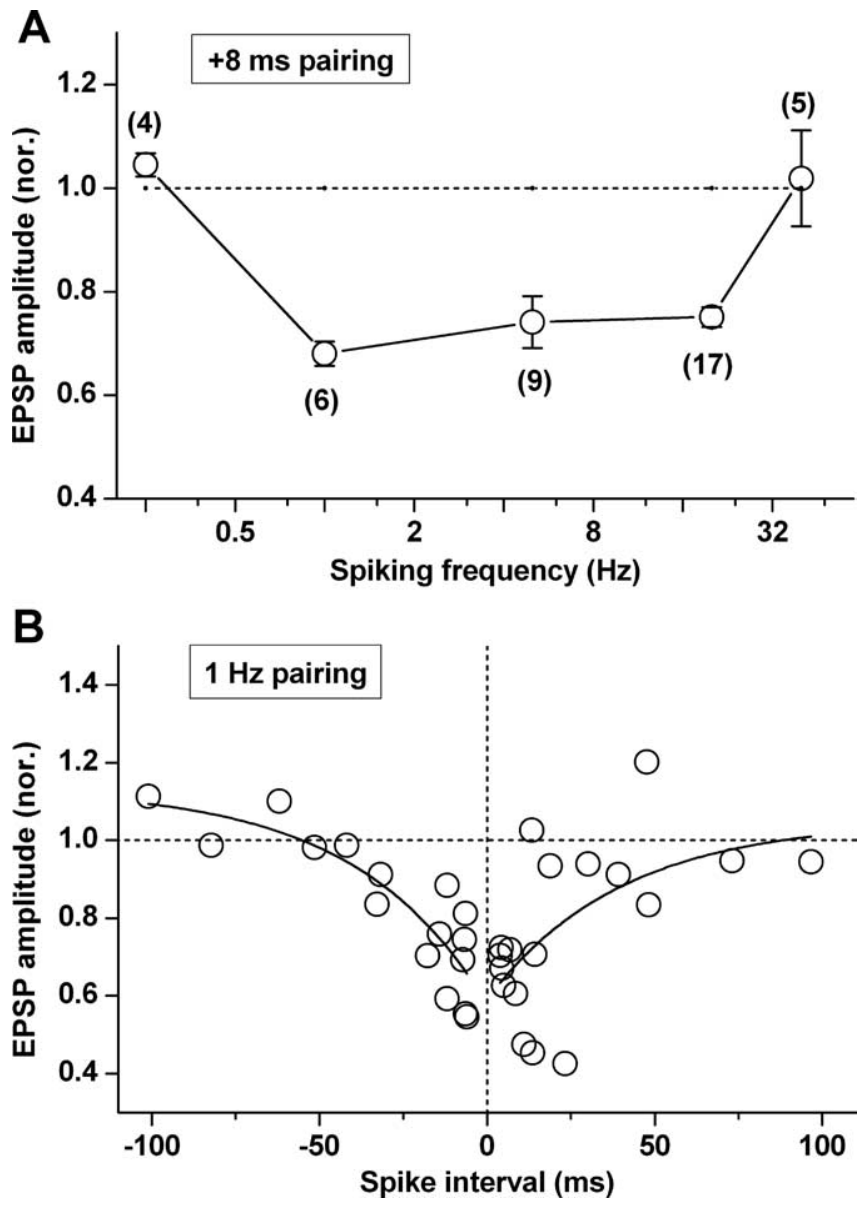

Figure 5. Spike-timing-dependent modification of PC-FS synapses under various frequency and timing of correlated spiking. $\boldsymbol{A}$, Dependence on the spiking frequency at the same interval of correlated pre-post spiking ( $+8 \mathrm{~ms}$ ). $\boldsymbol{B}$, Dependence on the time interval of presynaptic and postsynaptic spiking at the same frequency $(1 \mathrm{~Hz})$. Each point represents result from one cel pair. Curves, Single-exponential fit with $y=y_{0}+A \times e^{-x / \tau}[y$, normalized EPSP values; $x$, timing intervals; for pre-post pairing, $A=-0.46, \tau=39.9 \mathrm{~ms}(n=18)$; for post-pre pairing, $A=-0.56, \tau=39.1 \mathrm{~ms}(n=16)]$. Error bars depict SEM.

col (Fig. $7 A, B)$, suggesting common expression mechanism for LTD induced under these two different conditions. In contrast to results found at PC-LTS synapses, LTD induced by either +8 or $-8 \mathrm{~ms}$ spike pairing (at $20 \mathrm{~Hz}$ ) was not accompanied by any change in the CV of evoked EPSPs at PC-FS synapses (Fig. 7A, supplemental Fig. $1 B$, available at www.jneurosci.org as supplemental material), suggesting a different LTD expression mechanism.

Changes in the paired-pulse ratio (PPR) are also used to suggest presynaptic alteration in the transmitter release probability (Manabe et al., 1993; Neher, 1998; Bellingham and Walmsley, 1999), although a postsynaptic mechanism involving activitydependent removal of postsynaptic polyamine at PC-multipolar cell synapses can contribute to PPR changes (Rozov and Burnashev, 1999). We found that PPR at PC-LTS synapses showed bidirectional changes after LTP/LTD induction (Fig. 7C), with reduced PPR ( $79 \pm 8 \%$ of control; $n=11 ; p=0.012$ ) associated with LTP and increased PPR ( $145 \pm 15 \%$ of control; $n=8 ; p=$ 0.018 ) associated with LTD, consistent with presynaptic changes in the transmitter release at PC-LTS synapses. In contrast, no change in PPR was observed after LTD induction by either +8 or $-8 \mathrm{~ms}$ spike pairing at PC-FS synapses (96 $\pm 6 \%$ of the control; $n=34$ for $\pm 8 \mathrm{~ms}$ spike pairing at $20 \mathrm{~Hz} ; p=0.72$, paired $t$ test)
(Fig. 7C, supplemental Fig. $1 C$, available at www.jneurosci.org as supplemental material). Consistent with the findings on the changes in the $\mathrm{CV}$ and failure rate described above, these results again suggest LTD is expressed differently at PC-LTS and PC-FS synapses.

\section{Discussion}

Neocortical interneurons can be classified according to their electrophysiological properties, the presence of specific markers, and their synaptic location on the postsynaptic target cell (Markram et al., 2004). In the present study, we focused on two groups of layer 2/3 inhibitory interneurons, FS cells and LTS cells, which can be identified by their membrane properties, firing characteristics, and properties of innervated excitatory synapse. We observed a marked difference in STDP between PC-FS and PC-LTS synapses, in both the induction and the expression of spiketiming-dependent LTP/LTD.

\section{Target-cell-specific STDP at PC-interneuron synapses}

For the induction of STDP, we found that LTP and LTD can be induced at PC-LTS synapses by correlated spiking within a time interval of $<25 \mathrm{~ms}$, in a manner that depends on the temporal order of presynaptic and postsynaptic spiking. However, only LTD can be induced at PC-FS synapses, regardless of the order of spiking. Target-cell-dependent STDP has been observed in the dorsal cochlear nucleus of the brainstem, where parallel fiber (PF) synapses on excitatory fusiform principal neurons exhibit normal STDP, whereas only LTD can be induced at PF synapses on cartwheel inhibitory interneurons by pre-post spiking (Tzounopoulos et al., 2004). At the synapses between excitatory spiny neurons in layer 4 of rat barrel cortex, correlated spiking also induces only LTD (Egger et al., 1999). Activity-dependent longterm plasticity of excitatory synapses on inhibitory interneurons has been studied more extensively in the hippocampus (McMahon and Kauer, 1997; Cowan et al., 1998; Maccaferri et al., 1998; Laezza et al., 1999; Christie et al., 2000; Toth et al., 2000; Alle et al., 2001; Perez et al., 2001; Lei and McBain, 2002; Lamsa et al., 2005). However, the polarity of synaptic changes and underlying mechanisms remain controversial, presumably because of differences in the type of interneurons involved and in the experimental conditions used, including the LTP/LTD induction paradigms. Thus, although we did not observe LTP at layer 2/3 PC-FS synapses after correlated spiking, it remains possible that these synapses may exhibit LTP in response to other stimulation paradigms.

Target-cell-specific synaptic properties, including STDP, may arise from differences in the composition of postsynaptic receptors (Lopez-Bendito et al., 2002; Nyiri et al., 2003), properties of postsynaptic $\mathrm{Ca}^{2+}$ regulation (Goldberg et al., 2003; Kaiser et al., 2004), expression of receptors for retrograde signals in the axon terminals (Tzounopoulos et al., 2007), as well as presynaptic transmitter release machinery (Reyes et al., 1998; Koester and Johnston, 2005). Differential properties of different nerve terminals are already indicated by the differential transmitter release probability and short-term plasticity of PC synapses on multipolar and bitufted cells (Koester and Johnston, 2005). In the present study, we further showed that long-term plasticity, in the form of STDP, also exhibits target-cell specificity at PC-interneuron synapses. Differences in the short-term plasticity (e.g., synaptic facilitation/depression) may have resulted in differences in the efficacy of presynaptic activity in driving postsynaptic excitation, leading to target-cell-specific STDP. However, the latter possibility was excluded by our finding that target-cell-specific LTP was 
still observed when spike pairing was applied at a low frequency $(+8 \mathrm{~ms}$ at $1 \mathrm{~Hz})$ that did not induce any short-term facilitation/depression (Fig. 4).

Among many potential synaptic differences, we found that the level of NMDARs relative to non-NMDARs is much higher at PC-LTS synapses than that at PC-FS synapses (supplemental Fig. 3, available at www.jneurosci.org as supplemental material). This result is reminiscent of the findings that $\mathrm{Ca}^{2+}$ elevation in the dendrite of bitufted interneurons is NMDARdependent (Kaiser et al., 2004), and that $\mathrm{Ca}^{2+}$ signals at layer $2 / 3$ parvalbuminpositive FS cells is mediated by $\mathrm{Ca}^{2+}$ permeable AMPARs, but not by NMDARs (Goldberg et al., 2003). In rat motor cortex, bath application of AP5 also had a minor effect on the amplitude and duration of unitary EPSPs at layer 5 PC-FS synapses (Angulo et al., 1999). Thus, differential postsynaptic expression of NMDARs in various types of interneurons may contribute to target-cell-specific synaptic functions, including long-term plasticity. Whether and to what extent the absence of LTP at PC-FS synapse can be attributed to the lower relative level of NMDARs remains to be further elucidated.

\section{Cellular mechanisms underlying differential STDP}

It is generally accepted that the pattern of postsynaptic $\mathrm{Ca}^{2+}$ elevation plays a critical role in determining the polarity of synaptic plasticity. Elevation of $\mathrm{Ca}^{2+}$ below a threshold level has no effect on synaptic efficacy, an intermediate elevation of $\mathrm{Ca}^{2+}$ results in LTD, and highlevel $\mathrm{Ca}^{2+}$ transient induces LTP (Neveu and Zucker, 1996; Franks and Sejnowski, 2002; Malenka and Bear, 2004). At many synapses, $\mathrm{Ca}^{2+}$ elevation resulting from activation of NMDARs is required for the induction of both LTP and LTD (Malenka and Bear 2004), including spike-timing-dependent LTP/LTD (Bi and Poo, 1998; Dan and Poo, 2006), consistent with the notion that the NMDAR serves for as the coincidence detector and is required for both LTP and LTD. However, computational analyses have suggested that a second coincidence detector independent of NMDARs is necessary to specifically mediate spike-timingdependent LTD (Karmarkar and Buonomano, 2002). Indeed, previous experimental studies on excitatory synapses from layer 4 to layer 2/3 PCs in the somatosensory cortex showed that spiketiming-dependent LTD depends on the activation of both mGluR/ $\mathrm{IP}_{3} \mathrm{R}$ pathway and voltage-dependent $\mathrm{Ca}^{2+}$ channels (VDCCs), but not postsynaptic NMDARs (Bender et al., 2006). Moreover, the polarity of STDP at local excitatory inputs to layer $2 / 3$ PCs in the somatosensory cortex is not determined by the volume-averaged peak $\mathrm{Ca}^{2+}$ elevation in the postsynaptic spine, but by the timing of
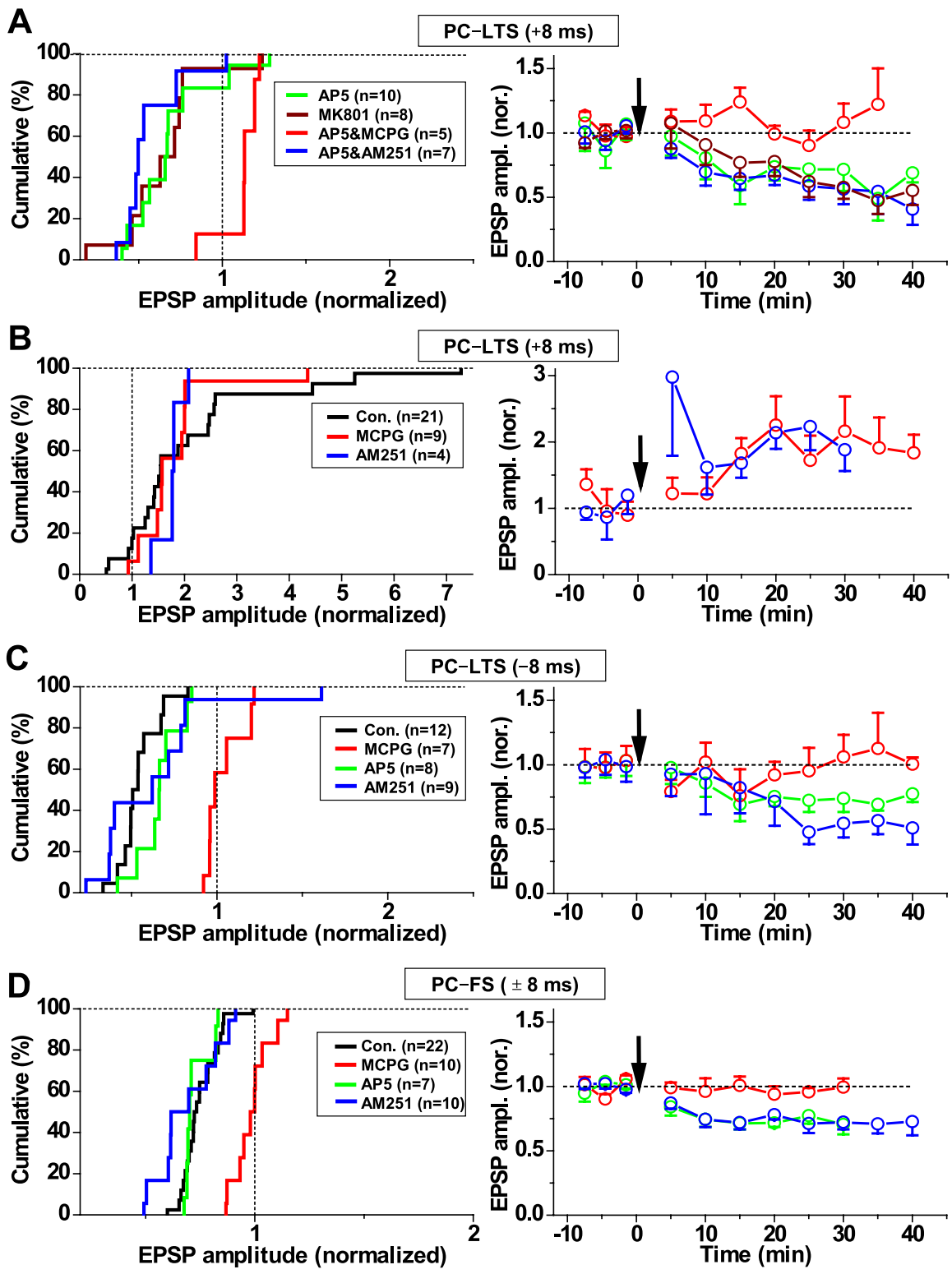

Figure 6. Distinct roles of NMDARs and mGluRs in spike-timing-dependent LTP/LTD of PC-LTS and PC-FS synapses. $A, B$, Left, Cumulative percentage plot for the distribution of the mean EPSP amplitude $10-60$ min after correlated spiking (at $20 \mathrm{~Hz}$ ) at an interval of $+8 \mathrm{~ms}$ at PC-LTS synapses, normalized by the mean amplitude before spiking, for experiments in the bath presence of Right, Time course of the average change in EPSP amplitude (over a $3 \mathrm{~min}$ bin in the baseline and over a $5 \mathrm{~min}$ bin after the pairing) before and after the $+8 \mathrm{~ms}$ pre-post spike pairing (at $20 \mathrm{~Hz}$ ) for the cells shown on the left. $C$, Similar to $A$ except that $-8 \mathrm{~ms}$ post-pre spike pairing (at $20 \mathrm{~Hz}$ ) was used at PC-LTS synapses. D, Similar to C except that the experiments were performed at PC-FS synapses. Error bars indicate SEM.

mGluR activation relative to that of spiking-induced VDCC activation (Nevian and Sakmann, 2006). Thus, in addition to the NMDAR, the mGluR now emerges as the second coincidence detector, serving specifically for the induction of spike-timingdependent LTD.

Our present results in neocortical PC-interneuronal synapses are consistent with the notion that NMDAR and mGluR serve as two independent coincidence detectors for the induction of LTP and LTD, respectively. After pre-post correlated spiking at PCLTS synapses, NMDAR-dependent LTP was induced. This LTP was not affected when mGluRs were blocked. However, when NMDARs were blocked, the same pre-post correlated spiking induced mGluR-dependent LTD. Thus, the activity of NMDARs 
A

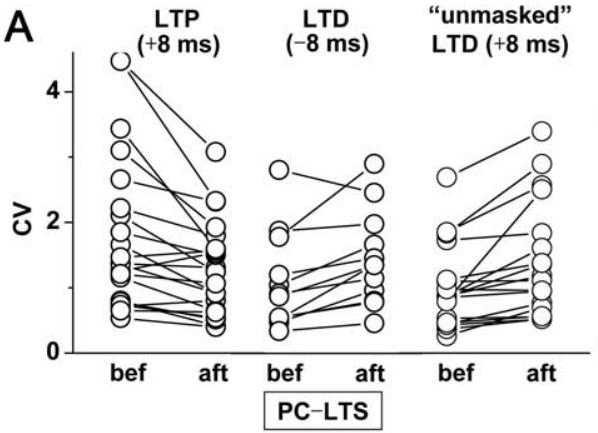

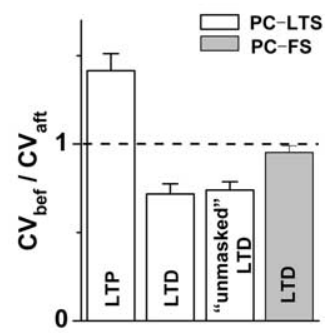

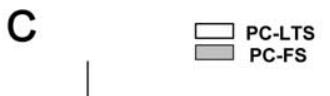

B

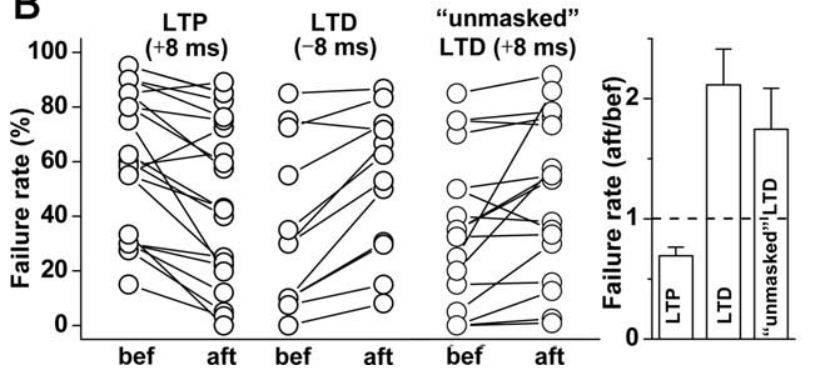

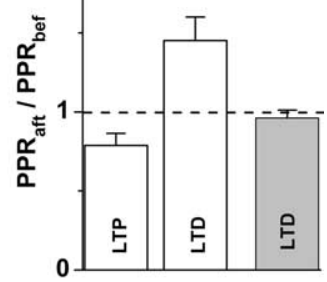

Figure 7. Distinct expression of STDP at PC-LTS and PC-FS synapses. $A$, Analysis of CV of evoked EPSPs showed changes at PC-LTS synapses, but not at PC-FS synapses after correlated spiking. CV $=(\sigma / m)$, where $\sigma$ and $m$ are the SD and the mean of EPSP amplitudes. The values of $\sigma$ and $m$ measured for a duration of 10 min immediately before and $20 \sim 30$ min after correlated spiking were used to calculate $\mathrm{CV}_{\text {bef }}$ and $\mathrm{CV}_{\text {aft' }}$ respectively. Each pair of connected circles represents mean $\mathrm{CV}_{\text {bef }}$ and $\mathrm{CV}_{\text {aft }}$ obtained from individual experiments shown in Figure 2, $C$ and $F$, and Figure 6 A. Unmasked LTD, LTD found at PC-LTS synapses in experiments with $+8 \mathrm{~ms}$ pairing in the presence of MK801 and AP5. A significant difference between $\mathrm{CV}_{\text {bef }}$ and $\mathrm{CV}_{\text {aft }}$ was observed at PC-LTS synapses (LTP of +8 ms pairing, $p=0.0013, n=21$; LTD of -8 ms pairing, $p=0.0065, n=12 ;$ unmasked LTD, $p=0.0013$, $n=18$; paired $t$ test), but not at $P(-F S$ synapses ( \pm 8 ms pairing, $p=0.33, n=41$, paired $t$ test). The histogram depicts the ratio of $\mathrm{CV}$, determined as $\mathrm{CV}_{\text {bef }} / \mathrm{CV}_{\text {aft }}$. $\boldsymbol{B}$, Change in the transmission failure rates at PC-LTS synapses accompanying LTP and LTD induction from experiments shown in Figure $2 C$, as well as unmasked LTD from experiments shown in Figure $6 A$. The failure rates were measured for $10 \mathrm{~min}$ before correlated spiking and for the duration from $10 \mathrm{~min}$ after spiking to end of the experiment, respectively. Data from the same cell pair were connected by a line in the left panels. Histograms depict the average values ( \pm SEM) of normalized failure rates (to mean value before the induction) for the data set shown on the left. $\boldsymbol{C}$, Analysis of PPR of evoked EPSPs showed bidirectional changes at PC-LTS synapses, but no change at PC-FS synapses after correlated spiking. The interval between paired pulses was $100 \mathrm{~ms}$. The value in PPR for individual cell pairs was measured in the same manner as in $\boldsymbol{A}$. The histogram depicts the ratio of PPR, determined as $P P R_{\text {aft }} / P P R_{\text {bef }}$.

appears to dominate over that of mGluRs and LTP induction causes a masking or inhibition of LTD induction at these synapses. It is possible that the high-level $\mathrm{Ca}^{2+}$ elevation resulting from NMDAR activation leads to LTP induction, regardless of the mGluR activity, whereas the latter is sufficient to trigger LTD when NMDARs are blocked

At neocortical PC-interneuron synapses, we found that the expression of LTD at PC-FS synapses is postsynaptic, whereas both LTP and LTD at PC-LTS synapses involve presynaptic modifications. Differential expression of STDP was also reported in the dorsal cochlear nucleus (Tzounopoulos et al., 2007). At PF synapses on fusiform principle cells, LTP is expressed postsynaptically. In contrast, at PF synapses on cartwheel interneurons, endocannabinoiddependent LTD is expressed presynaptically, although postsynaptically expressed LTP can be revealed when endocannabinoid receptors were blocked. Presynaptically expressed LTP and LTD often require retrograde signaling from the postsynaptic cells. The retrograde signaling may be mediated by postsynaptically derived diffusible factors (Tao and Poo, 2001) or trans-synaptic interactions between presynaptic and postsynaptic membrane-bound proteins (Futai et al., 2007). In addition to the endocannabinoid associated with spike-timing-dependent LTD (Sjostrom et al., 2003; Bender et al., 2006; Tzounopoulos et al., 2007), there is evidence for other retrograde factors associated with LTP/LTD, including nitric oxide
(Schuman and Madison, 1991; Mu and Poo, 2006; Sjostrom et al., 2007) and brainderived neurotrophic factor (Schinder et al., 2000; $\mathrm{Mu}$ and Poo, 2006). Given the fact that spike-timing-dependent LTP and LTD involve the presynaptic expression at PC-LTS synapses, two different types of retrograde factors may be involved in enhancing and reducing presynaptic transmitter release from $\mathrm{PC}$ axon terminals, respectively.

In conclusion, our data provide strong evidence that target-cell-dependent STDP exists at divergent output synapses of the $\mathrm{PC}$ on inhibitory interneurons in the neocortex. Modulation of interneuron excitation by PC synapses is a key factor for maintaining the balance between excitation and inhibition in cortical neuronal networks. The disparity in the axonal and dendritic morphology between LTS and FS cells and in reciprocal connections between PC-LTS and PC-FS connections found in this study suggests that these LTS and FS cells serve distinct network functions in the cortex. Differential strengthening and weakening of PC connections with these cells by the same PC spiking activity thus allow selective processing and storage of information in local cortical circuits.

\section{References}

Alle H, Jonas P, Geiger JR (2001) PTP and LTP at a hippocampal mossy fiber-interneuron synapse. Proc Natl Acad Sci USA 98:14708-14713.

Angulo MC, Rossier J, Audinat E (1999) Postsynaptic glutamate receptors and integrative properties of fast-spiking interneurons in the rat neocortex. J Neurophysiol 82:1295-1302.

Bacci A, Rudolph U, Huguenard JR, Prince DA (2003) Major differences in inhibitory synaptic transmission onto two neocortical interneuron subclasses J Neurosci 23:9664-9674.

Bacci A, Huguenard JR, Prince DA (2004) Long-lasting self-inhibition of neocortical interneurons mediated by endocannabinoids. Nature 431:312-316.

Bekkers JM, Stevens CF (1990) Presynaptic mechanism for long-term potentiation in the hippocampus. Nature 346:724-729.

Bellingham MC, Walmsley B (1999) A novel presynaptic inhibitory mechanism underlies paired pulse depression at a fast central synapse. Neuron 23:159-170.

Bender VA, Bender KJ, Brasier DJ, Feldman DE (2006) Two coincidence detectors for spike timing-dependent plasticity in somatosensory cortex. J Neurosci 26:4166-4177.

Bi GQ, Poo MM (1998) Synaptic modifications in cultured hippocampal neurons: dependence on spike timing, synaptic strength, and postsynaptic cell type. J Neurosci 18:10464-10472.

Bliss TV, Collingridge GL (1993) A synaptic model of memory: long-term potentiation in the hippocampus. Nature 361:31-39.

Christie BR, Franks KM, Seamans JK, Saga K, Sejnowski TJ (2000) Synaptic plasticity in morphologically identified CA1 stratum radiatum interneurons and giant projection cells. Hippocampus 10:673-683.

Cowan AI, Stricker C, Reece LJ, Redman SJ (1998) Long-term plasticity at excitatory synapses on aspinous interneurons in area CA1 lacks synaptic specificity. J Neurophysiol 79:13-20.

Dan Y, Poo MM (2006) Spike timing-dependent plasticity: from synapse to perception. Physiol Rev 86:1033-1048.

Debanne D, Gahwiler BH, Thompson SM (1998) Long-term synaptic plas- 
ticity between pairs of individual CA3 pyramidal cells in rat hippocampal slice cultures. J Physiol 507:237-247.

Egger V, Feldmeyer D, Sakmann B (1999) Coincidence detection and changes of synaptic efficacy in spiny stellate neurons in rat barrel cortex. Nat Neurosci 2:1098-1105.

Feldman DE (2000) Timing-based LTP and LTD at vertical inputs to layer II/III pyramidal cells in rat barrel cortex. Neuron 27:45-56.

Franks KM, Sejnowski TJ (2002) Complexity of calcium signaling in synaptic spines. Bioessays 24:1130-1144.

Froemke RC, Dan Y (2002) Spike-timing-dependent synaptic modification induced by natural spike trains. Nature 416:433-438.

Futai K, Kim MJ, Hashikawa T, Scheiffele, Sheng M, Hayashi Y (2007) Retrograde modulation of presynaptic release probability through signaling mediated by PSD-95-neuroligin. Nat Neurosci 10:186-195.

Goldberg JH, Tamas G, Aronov D, Yuste R (2003) Calcium microdomains in aspiny dendrites. Neuron 40:807-821.

Goodman CS, Shatz CJ (1993) Developmental mechanisms that generate precise patterns of neuronal connectivity. Cell 72 [Suppl]:77-98.

Kaiser KM, Lubke J, Zilberter Y, Sakmann B (2004) Postsynaptic calcium influx at single synaptic contacts between pyramidal neurons and bitufted interneurons in layer $2 / 3$ of rat neocortex is enhanced by backpropagating action potentials. J Neurosci 24:1319-1329.

Karmarkar U, Buonomano DV (2002) A model of spike-timing dependent plasticity: one or two coincidence detector? J Neurophysiol 88:507-513.

Kawaguchi Y, Kubota Y (1997) GABAergic cell subtypes and their synaptic connections in rat frontal cortex. Cereb Cortex 7:476-486.

Koester HJ, Johnston D (2005) Target cell-dependent normalization of transmitter release at neocortical synapses. Science 308:863-866.

Laezza F, Doherty JJ, Dingledine R (1999) Long-term depression in hippocampal interneurons: joint requirement for pre- and postsynaptic events. Science 285:1411-1414.

Lamsa K, Heeroma JH, Kullmann DM (2005) Hebbian LTP in feed-forward inhibitory interneurons and the temporal fidelity of input discrimination. Nat Neurosci 8:916-924.

Lei S, McBain CJ (2002) Distinct NMDA receptors provide differential modes of transmission at mossy fiber-interneuron synapses. Neuron 33:921-933.

Liao D, Hessler NA, Malinow R (1995) Activation of postsynaptically silent synapses during pairing-induced LTP in CA1 region of hippocampal slice. Nature 375:400-404.

Lopez-Bendito G, Shigemoto R, Fairen A, Lujan R (2002) Differential distribution of group I metabotropic glutamate receptors during rat cortical development. Cereb Cortex 12:625-638.

Maccaferri G, Toth K, McBain CJ (1998) Target-specific expression of presynaptic mossy fiber plasticity. Science 279:1368-1370.

Malenka RC, Bear MF (2004) LTP and LTD: an embarrassment of riches. Neuron 44:5-21.

Malinow R, Tsien RW (1990) Presynaptic enhancement shown by wholecell recordings of long-term potentiation in hippocampal slices. Nature 346:177-180.

Manabe T, Wyllie DJ, Perkel DJ, Nicoll RA (1993) Modulation of synaptic transmission and long-term potentiation: effects on paired pulse facilitation and EPSC variance in the CA1 region of the hippocampus. J Neurophysiol 70:1451-1459.

Markram H, Lubke J, Frotscher M, Sakmann B (1997) Regulation of synaptic efficacy by coincidence of postsynaptic APs and EPSPs. Science 275:213-215.

Markram H, Toledo-Rodriguez M, Wang Y, Gupta A, Silberberg G, Wu C (2004) Interneurons of the neocortical inhibitory system. Nat Rev Neurosci 5:793-807.
Martin SJ, Grimwood PD, Morris RG (2000) Synaptic plasticity and memory: an evaluation of the hypothesis. Annu Rev Neurosci 23:649-711.

McMahon LL, Kauer JA (1997) Hippocampal interneurons express a novel form of synaptic plasticity. Neuron 18:295-305.

Mu Y, Poo MM (2006) Spike timing-dependent LTP/LTD mediates visual experience-dependent plasticity in a developing retinotectal system. Neuron 50:115-125.

Neher E (1998) Vesicle pools and $\mathrm{Ca}^{2+}$ microdomains: new tools for understanding their roles in neurotransmitter release. Neuron 20:389-399.

Neveu D, Zucker RS (1996) Postsynaptic levels of $\left[\mathrm{Ca}^{2+}\right]_{i}$ needed to trigger LTD and LTP. Neuron 16:619-629.

Nevian T, Sakmann B (2006) Spine $\mathrm{Ca}^{2+}$ signaling in spike-timingdependent plasticity. J Neurosci 26:11001-11013.

Nyiri G, Stephenson FA, Freund TF, Somogyi P (2003) Large variability in synaptic $\mathrm{N}$-methyl-D-aspartate receptor density on interneurons and a comparison with pyramidal-cell spines in the rat hippocampus. Neuroscience 119:347-363.

Perez Y, Morin F, Lacaille JC (2001) A hebbian form of long-term potentiation dependent on mGluR1a in hippocampal inhibitory interneurons. Proc Natl Acad Sci USA 98:9401-9406.

Reyes A, Lujan R, Rozov A, Burnashev N, Somogyi P, Sakmann B (1998) Target-cell-specific facilitation and depression in neocortical circuits. Nat Neurosci 1:279-285.

Rozov A, Burnashev N (1999) Polyamine-dependent facilitation of postsynaptic AMPA receptors counteracts paired-pulse depression. Nature 401:594-598.

Sakmann B, von Kitzing E, Roth A (1998) Unitary EPSPs in pairs of anatomically reconstructed cortical pyramidal neurons. In: Central synapses: quantal mechanisms and plasticity (Faber DS, Korn H, Redman S, Thompson SM, Altman JS, eds), pp 64-73. Strasbourg: Human Frontier Science Program.

Schinder AF, Berninger B, Poo M (2000) Postsynaptic target specificity of neurotrophin-induced presynaptic potentiation. Neuron 25:151-163.

Schuman EM, Madison DV (1991) A requirement for the intercellular messenger nitric oxide in long-term potentiation. Science 254:1503-1506.

Sjostrom PJ, Turrigiano GG, Nelson SB (2001) Rate, timing, and cooperativity jointly determine cortical synaptic plasticity. Neuron 32:1149-1164.

Sjostrom PJ, Turrigiano GG, Nelson SB (2003) Neocortical LTD via coincident activation of presynaptic NMDA and cannabinoid receptors. Neuron 39:641-654.

Sjostrom PJ, Turrigiano GG, Nelson SB (2007) Multiple forms of long-term plasticity at unitary neocortical layer 5 synapses. Neuropharmacology $52: 176-184$.

Song S, Abbott LF (2001) Cortical development and remapping through spike timing-dependent plasticity. Neuron 32:339-350.

Tao H, Poo MM (2001) Retrograde signaling at central synapses. Proc Natl Acad Sci USA 98:11009-11015.

Toth K, Suares G, Lawrence JJ, Philips-Tansey E, McBain CJ (2000) Differential mechanisms of transmission at three types of mossy fiber synapse. J Neurosci 20:8279-8289.

Tzounopoulos T, Kim Y, Oertel D, Trussell LO (2004) Cell-specific, spike timing-dependent plasticities in the dorsal cochlear nucleus. Nat Neurosci 7:719-725.

Tzounopoulos T, Rubio ME, Keen JE, Trussell LO (2007) Coactivation of pre- and postsynaptic signaling mechanisms determines cell-specific spike-timing-dependent plasticity. Neuron 54:291-301.

Zhang LI, Poo MM (2001) Electrical activity and development of neural circuits. Nat Neurosci 4 [Suppl]:1207-1214.

Zucker RS, Regehr WG (2002) Short-term synaptic plasticity. Annu Rev Physiol 64:355-405. 